\title{
Association between dietary patterns and chronic kidney disease in a middle-aged Chinese population
}

\author{
Shan-Shan $\mathrm{Xu}^{1}$, Jun Hua ${ }^{2}, \mathrm{Yi}$-Qian Huang ${ }^{3}$ and Long Shu ${ }^{4, *}$ \\ 'School of Medicine, Huainan Union University, Huainan, Anhui, People's Republic of China: ${ }^{2}$ Department of \\ Neurology, General Hospital of Huainan Eastern Hospital, Huainan, Anhui, People's Republic of China: ${ }^{3}$ Department \\ of Digestion, Zhejiang Hospital, Hangzhou, Zhejiang, People's Republic of China: ${ }^{4}$ Department of Nutrition, Zhejiang \\ Hospital, Xihu District, Hangzhou 310013, Zhejiang, People's Republic of China
}

Submitted 29 January 2019: Final revision received 14 June 2019: Accepted 19 June 2019: First published online 2 0ctober 2019

\begin{abstract}
Objective: To explore the relationship between dietary patterns and risk of chronic kidney disease (CKD) in Chinese adults aged 45-59 years.

Design: Dietary data were collected using a semi-quantitative FFQ. Factor analysis was used to identify the major dietary patterns. Logistic regression models were applied to clarify the association between dietary patterns and the risk of CKD. Setting: The present study population was a part of the population-based Nutrition and Health Study performed in the city of Hangzhou, Zhejiang Province, eastern China.

Participants: A total of 2437 eligible participants (45-59 years) were enrolled in the present cross-sectional study from June 2015 to December 2016.

Results: Three major dietary patterns were identified: 'traditional southern Chinese', 'Western' and 'grains-vegetables' patterns, collectively accounting for $25.6 \%$ of variance in the diet. After adjustment for potential confounders, participants in the highest quartile of the Western pattern had greater odds for CKD (OR $=1.83,95 \%$ CI $1.21,2.81 ; P<0.05)$ than those in the lowest quartile. Compared with the lowest quartile of the grains-vegetables pattern, the highest quartile had lower odds for CKD (OR $=0.84,95 \%$ CI $0.77,0.93 ; P<0.05)$. In addition, there was no significant association between the traditional southern Chinese pattern and risk of CKD $(P>0.05)$.

Conclusions: Our results suggest that the Western pattern is associated with an increased risk, whereas the grains-vegetables pattern is associated with a reduced risk for CKD. These findings can guide dietary interventions for the prevention of CKD in a middle-aged Chinese population.
\end{abstract}

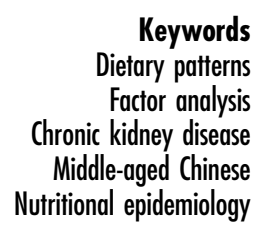

Chronic kidney disease (CKD) is recognized a public health problem with poor clinical outcomes, affecting $8-16 \%$ of the population worldwide ${ }^{(1)}$. The burden of CKD has implications for the demand for renal replacement therapy and is associated with increased risks of morbidity, mortality and hospitalization $^{(1,2)}$. In the USA, CKD is a common chronic disease, with an estimated 26 million adults affected in $2007^{(3)}$. In Taiwan, the average prevalence of CKD was $11.9 \%$ from 1994 to 2006 , and it was higher in patients with low socio-economic status $(19.9 \%)^{(4)}$. Zhang et al. reported that the overall prevalence of CKD surpassed 119.5 million (approximately $10.8 \%$ of the general population) in a nationally representative sample of Chinese adults ${ }^{(5)}$. Known risk factors for CKD include obesity, hypertension, diabetes, smoking and use of nephrotoxic medications ${ }^{(1)}$. Moreover, dietary factors have also been recognized as important risk factors for $\mathrm{CKD}^{(6)}$. For example, high intakes of refined grains, high-fat dairy, red meat, processed meat and refined sugars are associated with increased risk of $\mathrm{CKD}^{(7)}$.

In recent decades, many epidemiological studies have focused on diet as an important pathogenic factor for the development of $\mathrm{CKD}^{(7)}$. Many of these studies have focused on the effects of individual nutrients or foods and food groups ${ }^{(8-10)}$. However, in reality, people generally consume a combination of various foods and nutrients in each meal, not individual foods and nutrients. Besides, individual foods and nutrients do not show the interactive 
and synergistic effects of different foods and nutrients in a whole $\operatorname{diet}^{(11)}$. In this context, dietary pattern analysis has emerged in nutritional epidemiology as an alternative approach for assessing the association between diet and chronic non-communicable diseases, since it considers the complexity of the overall diet and may provide guidance for nutrition intervention and education ${ }^{(12)}$.

Several past studies have explored the association between dietary pattern and CKD risk ${ }^{(6,13-18)}$. However, almost all of these studies have been conducted in Western and Korean populations ${ }^{(13,15-18)}$, while research in the Chinese population has been very limited ${ }^{(14)}$. Only one published study has investigated dietary patterns in relation to $\mathrm{CKD}$ risk in the Chinese population ${ }^{(14)}$. Furthermore, to the authors' knowledge, no previous study has assessed the association between overall dietary patterns and the risk of CKD among a middle-aged Chinese population. To fill such gaps in the literature, the present study aimed to identify the major dietary patterns existing in a middle-aged Chinese population and to evaluate their association with the risk of CKD.

\section{Materials and methods}

\section{Study population}

The present cross-sectional study was conducted in Hangzhou city, the capital of Zhejiang Province, eastern China, from June 2015 to December 2016. The details of the study and the recruitment procedures are described elsewhere $^{(12)}$. The sample was taken from ten areas (Xihu, Gongshu, Shangcheng, Xiacheng, Bingjiang, Jianggan, Xiaoshan, Yuhang, Fuyang and Linan) and three counties (Tonglu, Chunan and Jiande) by a stratified cluster random-sampling method. We chose two residential villages or communities from every county or area randomly, according to resident health records, with participants being individuals aged 45-59 years residing in the selected villages or communities. A total of 2437 eligible participants (1143 males, 1294 females; aged between 45 and 59 years) were invited to attend a health examination at the Medical Center for Physical Examination, Zhejiang Hospital. Each individual participated in a face-to-face interview with a trained interviewer using written questionnaires. Of the 2437 participants in the original study, we excluded 213 participants who reported implausible energy intakes $(<2510$ or $>16736 \mathrm{~kJ}$ ( $<600$ or $>4000 \mathrm{kcal}$ for males); $<2092$ or $>12552 \mathrm{~kJ}$ ( $<500$ or $>3000 \mathrm{kcal}$ ) for females). Additionally, twenty-five participants were excluded due to missing serum creatinine or urine albumin values; and 195 were excluded because of incomplete anthropometric information and/or missing or incomplete information on their dietary intake. This resulted in a total of 2004 participants (1003 males and 1001 females) for inclusion in the present study.

\section{Assessment of dietary intake}

Dietary intake was assessed using a validated semiquantitative FFQ containing 138 food items. This FFQ was based on the FFQ used in the 2010 China National Nutrition and Health Survey (CNNHS), which has been validated among a middle-aged Chinese population ${ }^{(12)}$. Before that study, a pilot survey on the validity of the FFQ was performed in this population by comparison with three $24 \mathrm{~h}$ dietary recalls. Thus, the validity and reliability of this FFQ in terms of food consumption have been documented elsewhere $^{(12)}$. Participants were asked to recall the consumption frequency of each food item in the previous 12 months (never, less than 1 time/month, 1-3 times/ month, 1-2 times/week, 3-4 times/week, 5-6 times/week, 1 time/d, 2 times/d, 3 times/d) and the estimated portion size, using local weight units ( 1 Liang $=50 \mathrm{~g}$ ) or natural units (cups). Data from the FFQ were then converted to grams or millilitres per day and used in the following analyses.

\section{Identification of dietary patterns}

Individual food items from this FFQ were aggregated into thirty predefined food groups based on similarity of type of food and nutrient composition (see online supplementary material, Supplemental Table S1). The KaiserMeyer-Olkin measure of sample adequacy and Bartlett's test of sphericity were applied to assess the sample adequacy for factor analysis. Subsequently, we conducted factor analysis (principal component) to derive major dietary patterns. The number of factors retained was based on the following criteria: components with an eigenvalue $\geq 2 \cdot 0$; scree plot test; and interpretability of the factors. The factors identified were rotated by orthogonal transformation (varimax rotation), which maintains the uncorrelated factors and obtains a simpler structure with greater interpretability ${ }^{(19)}$. Food groups with a factor loading $\geq|0.4|$ were considered to be the major contributors to each pattern.

The labelling of dietary patterns was based on the interpretation of foods with high factor loadings on each pattern $^{(20)}$. The factor scores for each dietary pattern were calculated by summing observed intakes of food groups weighted by their factor loadings. In our analyses, factor scores for each pattern were categorized into quartiles (Q1 represents a low intake of the food pattern; Q4 represents a high intake of the food pattern). The first quartile of each pattern was used as the reference group.

\section{Assessment of anthropometric variables}

Body height and weight were measured with participants standing without shoes and wearing light clothing. Height was measured to the nearest $0 \cdot 1 \mathrm{~cm}$ and weight was measured to the nearest $0 \cdot 1 \mathrm{~kg}$ using a balance-beam scale. BMI was calculated as weight in kilograms divided by the square of height in metres. Waist circumference (WC) was measured at the midpoint between the lower rib edge 
and the upper iliac crest by means of a metric measure with an accuracy of $1 \mathrm{~mm}$, and hip circumference was measured at the maximum level over light clothing by using an inelastic plastic tape ${ }^{(21)}$. All measurements were carried out by trained researchers.

\section{Assessment of other variables}

Information on physical activity was collected using the International Physical Activity Questionnaire (IPAQ) ${ }^{(22)}$ and expressed as metabolic equivalent hours per week $($ MET $\times \mathrm{h} /$ week $)$, in which different activities were ranked on a scale from sleeping (0.9 MET) to high-intensity physical activities ( $>6 \mathrm{MET}$ ). The questionnaire also collected data on participants' smoking habit and level of education. Smoking data classified participants as 'never smokers', 'former smokers' and 'current smokers'. Educational level was classified into three categories (<high school (primary school or below); high school (middle and high school); > high school (junior college or above)). Total dietary energy intake for each participant was estimated through the semi-quantitative FFQ and expressed as kilocalories per day.

\section{Assessment of biomarkers}

Blood samples were obtained from participants between 07.00 and 09.00 hours after an overnight fast (12 h) and stored temporarily at $-20^{\circ} \mathrm{C}$ until analysis. After clotting, serum was separated by centrifugation for $15 \mathrm{~min}$ at $3000 \mathrm{rpm}$. The samples were then analysed in the Medical Center for Physical Examination, Zhejiang Hospital for fasting plasma glucose, total cholesterol, TAG, HDL-cholesterol, LDL-cholesterol, serum uric acid, serum creatinine, alanine aminotransferase and aspartate aminotransferase using the Hitachi 7180 automatic biochemical analyser (Tokyo, Japan).

\section{Assessment of blood pressure}

Each participant's resting blood pressure (resting in a seated position for at least $5 \mathrm{~min}$ ) was taken twice by a trained nurse using a standard mercury sphygmomanometer. The mean of the two measurements was utilized as the final blood pressure value for the study ${ }^{(23)}$.

\section{Definition of variables}

The CKD Epidemiology Collaboration (CKD-EPI) equation was used to estimate glomerular filtration rate (eGFR, in $\mathrm{ml} / \mathrm{min}$ per $1.73 \mathrm{~m}^{2}$ ); those with eGFR $<60 \mathrm{ml} / \mathrm{min}$ per $1.73 \mathrm{~m}^{2}$ or with the presence of albuminuria were classified as having $\mathrm{CKD}^{(24)}$. Serum creatinine was measured using Jaffe's kinetic methods. Urinary albumin was measured by the immunoturbidimetric method in a spot morning urine sample. Hypertension was defined as systolic blood pressure $\geq 140 \mathrm{mmHg}$ and/or diastolic blood pressure $\geq 90 \mathrm{mmHg}$, or the use of antihypertensive medication ${ }^{(23)}$. Obesity was defined as BMI $\geq 28 \mathrm{~kg} / \mathrm{m}^{2(25)}$ and abdominal obesity was defined as WC $\geq 85 \mathrm{~cm}$ for males and WC $\geq 80 \mathrm{~cm}$ for females in the Chinese population ${ }^{(26)}$.

\section{Statistical analyses}

Data were calculated across quartiles of each dietary pattern score, and presented as mean and standard deviation for continuous variables or as number and percentage for categorical variables. If data were normally distributed variables, we used the independent-samples $t$ test to assess the significant differences in continuous variables. If not, the Mann-Whitney $U$ test was applied. A $\chi^{2}$ test was also used to examine the difference between categorical variables. Logistic regression analysis models were used to examine the associations between dietary patterns and the risk of CKD with adjustment of potential confounding variables. In model 1, we adjusted for age (continuous) and gender. In model 2 , we additionally adjusted for income (continuous), education level (<high school, high school, $>$ high school), physical activity level (light, moderate, heavy), smoking status (never, current, former), BMI (continuous), type 2 diabetes mellitus (T2DM; yes/no) and hypertension (yes/no). In model 3, we further adjusted for total energy intake (continuous). All statistical analyses were performed using the statistical software package IBM SPSS Statistics version 22.0 and a two-sided $P<0.05$ was considered statistically significant.

\section{Results}

The overall prevalence of CKD in our study population was $7 \cdot 3 \%$. The demographic characteristics of participants based on CKD status are shown in Table 1 ( $n$ 2004). There were significant differences between participants with and without CKD by age, gender, smoking status, education, monthly income and the prevalence of obesity, hypertension and T2DM $(P<0.05)$.

The Kaiser-Meyer-Olkin index in the present study was $0 \cdot 704$, with a Bartlett's test value of $P<0.001$. These results indicated that the correlation between different dietary variables was strong enough for performing a factor analysis $^{(16)}$.

Three distinct dietary patterns were identified by factor analysis. These were labelled as: the 'traditional southern Chinese' pattern (higher intakes of refined grains, vegetables, fruits, pickled vegetables, fish and shrimp, bacon and salted fish, salted and preserved eggs, milk, soyabean and its products, miscellaneous bean, fats, drinks); the 'Western' pattern (higher intakes of red meats, poultry and organs, processed and cooked meat, eggs, seafood, cheese, fast foods, snacks, chocolates, alcoholic beverages, coffee); and the 'grains-vegetables' pattern (higher intakes of whole grains, tubers, vegetables, mushrooms, vegetable oil, nuts, honey, tea). These patterns explained 10.3, 8.5 and $6.8 \%$ of the dietary intake variance, respectively. 
Dietary patterns and chronic kidney disease

1061

Table 1 Demographic characteristics of the study participants by chronic kidney disease (CKD) status: Chinese adults aged 45-59 years ( $n$ 2004) from Hangzhou, Zhejiang Province, eastern China, June 2015-December 2016

\begin{tabular}{|c|c|c|c|c|c|}
\hline \multirow[b]{2}{*}{ Variable } & \multicolumn{2}{|c|}{$\begin{array}{l}\text { Participants with CKD } \\
\qquad(n 146)\end{array}$} & \multicolumn{2}{|c|}{$\begin{array}{l}\text { Participants without CKD } \\
\qquad(n \text { 1858) }\end{array}$} & \multirow[b]{2}{*}{ Significance* $^{\star}$} \\
\hline & Mean or $n$ & SD or $\%$ & Mean or $n$ & SD or $\%$ & \\
\hline Age (years) & $53 \cdot 7$ & $4 \cdot 3$ & $48 \cdot 5$ & 3.5 & $P<0.001$ \\
\hline \multicolumn{6}{|l|}{ Gender } \\
\hline Male & 59 & $40 \cdot 4$ & 944 & $50 \cdot 8$ & \multirow{2}{*}{$\begin{aligned} \chi^{2} & =5.85 \\
P & <0.05\end{aligned}$} \\
\hline Female & 87 & $59 \cdot 6$ & 914 & $49 \cdot 2$ & \\
\hline \multicolumn{6}{|l|}{ Smoking status } \\
\hline Never & 115 & $78 \cdot 8$ & 1499 & $80 \cdot 7$ & \multirow{3}{*}{$\begin{array}{r}\chi^{2}=13.81 \\
P<0.01\end{array}$} \\
\hline Former & 12 & $8 \cdot 2$ & 43 & $2 \cdot 3$ & \\
\hline Current & 19 & $13 \cdot 0$ & 316 & $17 \cdot 0$ & \\
\hline \multicolumn{6}{|l|}{ Education } \\
\hline$<$ High school & 25 & $17 \cdot 1$ & 167 & $9 \cdot 0$ & \multirow{3}{*}{$\begin{array}{r}\chi^{2}=13.90 \\
P<0.01\end{array}$} \\
\hline High school & 103 & $70 \cdot 6$ & 1310 & 70.5 & \\
\hline >High school & 18 & $12 \cdot 3$ & 381 & 20.5 & \\
\hline \multicolumn{6}{|c|}{ Monthly income per person (RMB) } \\
\hline$\leq 2500$ & 73 & $50 \cdot 0$ & 641 & 34.5 & \multirow{3}{*}{$\begin{array}{r}\chi^{2}=14.67 \\
P<0.01\end{array}$} \\
\hline $2500-4000$ & 60 & $41 \cdot 1$ & 953 & $51 \cdot 3$ & \\
\hline$>4000$ & 13 & 8.9 & 264 & $14 \cdot 2$ & \\
\hline \multicolumn{6}{|l|}{ Obese } \\
\hline Yes & 45 & $30 \cdot 8$ & 319 & $17 \cdot 2$ & \multirow{2}{*}{$\begin{aligned} \chi^{2} & =16.98 \\
P & <0.001\end{aligned}$} \\
\hline No & 101 & $69 \cdot 2$ & 1539 & $82 \cdot 8$ & \\
\hline \multicolumn{6}{|l|}{ Hypertension } \\
\hline Yes & 47 & $32 \cdot 2$ & 240 & $12 \cdot 9$ & \multirow{2}{*}{$\begin{aligned} \chi^{2} & =40.98 \\
P & <0.001\end{aligned}$} \\
\hline No & 99 & $67 \cdot 8$ & 1618 & $87 \cdot 1$ & \\
\hline \multicolumn{6}{|c|}{ Type 2 diabetes mellitus } \\
\hline Yes & 30 & 20.5 & 182 & $9 \cdot 8$ & \multirow{2}{*}{$\begin{aligned} \chi^{2} & =16.54 \\
P & <0.001\end{aligned}$} \\
\hline No & 116 & 79.5 & 1676 & $90 \cdot 2$ & \\
\hline
\end{tabular}

The factor loading matrices for the dietary patterns are provided in Table 2 .

The characteristics of the study participants by quartiles of dietary pattern scores are shown in Table 3. Participants within the top quartile of the traditional southern Chinese pattern were older, smokers and had a higher prevalence of CKD compared with participants in the lowest quartile. Compared with those in the lowest quartile, participants in the highest quartile of the Western pattern were younger, male, smokers, undertook light physical activity, had higher BMI, WC, waist-to-hip ratio and monthly income, and had higher prevalence of obesity, hypertension and CKD. Furthermore, we also found that participants in the highest quartile of the grains-vegetables pattern were older, female, never smokers, with lower BMI, WC, waist-to-hip ratio and total energy intake, had lower prevalence of obesity, hypertension and CKD, and had higher monthly income and physical activity level than those in the lowest quartile.

The major food consumption of the study participants by quartiles of dietary pattern scores is shown in Table 4. Compared with participants in the lowest quartile, those in the highest quartile of the traditional southern Chinese dietary pattern had higher intakes of refined grains, tubers, fruits, fish and shrimp, and eggs. In comparison with the participants from the lowest quartile of the Western dietary pattern, those in the highest quartile had higher intakes of refined grains, red meat, fish and shrimp, and drinks, and lower intake of whole grains. There were also significant differences in the consumption of whole grains, tubers, vegetables, fruits, red meat, fish and shrimp, eggs and drinks across quartiles of the grains-vegetables dietary pattern $(P<0.05)$.

The associations between dietary patterns and the risk of CKD by logistic regression analysis are presented in Table 5. After adjusting for potential confounding variables, participants in the highest quartile of the Western dietary pattern had greater odds of CKD (OR $=1.83,95 \%$ CI $1.21,2 \cdot 81 ; P<0.05)$ than those in the lowest quartile; while those in the highest quartile of the grains-vegetables dietary pattern had lower odds of CKD (OR $=0.84,95 \%$ CI $0.77,0.93 ; P<0.05)$ than those in the lowest quartile. In addition, we observed no significant association between the traditional southern Chinese pattern and the risk of CKD $(P>0.05)$.

\section{Discussion}

In our study of a Chinese population aged $45-59$ years, the prevalence of CKD was $7.3 \%$ and we identified three major dietary patterns in the population: traditional southern Chinese, Western and grains-vegetables patterns. The main findings were that the Western dietary pattern was 
Table 2 Factor loading matrix for the three dietary patterns* found in the study participants: Chinese adults aged $45-59$ years ( $n$ 2004) from Hangzhou, Zhejiang Province, eastern China, June 2015-December 2016

\begin{tabular}{|c|c|c|c|}
\hline \multirow[b]{2}{*}{ Food group } & \multicolumn{3}{|c|}{ Dietary pattern } \\
\hline & $\begin{array}{c}\text { Traditional } \\
\text { southern Chinese }\end{array}$ & Western & $\begin{array}{c}\text { Grains- } \\
\text { vegetables }\end{array}$ \\
\hline Refined grains & 0.41 & - & - \\
\hline Whole grains & - & - & 0.53 \\
\hline Tubers & - & - & 0.47 \\
\hline Vegetables & 0.41 & - & 0.64 \\
\hline Fruit & 0.46 & - & - \\
\hline Pickled vegetables & 0.51 & - & - \\
\hline Mushrooms & - & - & 0.66 \\
\hline Red meat & - & 0.56 & - \\
\hline Poultry and organs & - & 0.50 & - \\
\hline $\begin{array}{l}\text { Processed and } \\
\text { cooked meat }\end{array}$ & - & 0.52 & - \\
\hline Fish and shrimp & 0.49 & - & - \\
\hline Eggs & - & 0.35 & - \\
\hline Seafood & - & 0.42 & - \\
\hline Bacon and salted fish & 0.53 & - & - \\
\hline $\begin{array}{l}\text { Salted and } \\
\text { preserved eggs }\end{array}$ & 0.41 & - & - \\
\hline Milk & 0.36 & - & \\
\hline Cheese & - & 0.32 & \\
\hline $\begin{array}{l}\text { Soyabean and its } \\
\text { products }\end{array}$ & 0.40 & - & - \\
\hline Miscellaneous bean & 0.41 & - & - \\
\hline Fats & 0.45 & - & \\
\hline Vegetable oil & & - & 0.39 \\
\hline Fast foods & - & 0.41 & - \\
\hline Nuts & - & - & 0.30 \\
\hline Snacks & - & 0.52 & - \\
\hline Chocolates & - & 0.44 & - \\
\hline Honey & - & - & 0.60 \\
\hline Drinks & 0.47 & - & \\
\hline Alcoholic beverages & - & 0.30 & - \\
\hline Tea & - & - & 0.36 \\
\hline Coffee & - & 0.39 & - \\
\hline $\begin{array}{l}\text { Variance of intake } \\
\text { explained (\%) }\end{array}$ & $10 \cdot 3$ & $8 \cdot 5$ & $6 \cdot 8$ \\
\hline
\end{tabular}

*Absolute values $<0.4$ were excluded for simplicity.

positively associated with the risk of CKD, whereas the grains-vegetables dietary pattern was inversely associated with the risk of CKD. To the best of our knowledge, the present study is the first to examine the relationship of major dietary patterns with the risk of CKD in a middleaged Chinese population.

It is well known that CKD is a multifactorial chronic disease that may be associated with some aetiologies, including diabetes, obesity, hypertension and dietary factors $^{(1,6)}$. In our previous study, findings suggested that higher consumption of whole grains, tubers and vegetables is likely beneficial for the prevention of T2DM, while the higher consumption of red meats, poultry and organs, processed and cooked meat may increase the risk of $\mathrm{T}_{2} \mathrm{DM}^{(12)}$. In China, diabetes is one of the main contributors to $\mathrm{CKD}^{(27)}$. Besides, a substantial body of evidence has also demonstrated that diet plays a key role in the development of obesity and hypertension ${ }^{(28,29)}$. Jha et al. reported that hypertension and diabetes are the leading causes of CKD worldwide $^{(1)}$. In statistical analyses, there are two main approaches (a priori approach and a posteriori approach) to define dietary patterns. The a priori approach to evaluate dietary patterns uses previously defined scores to assess the overall diet quality, based on prevailing dietary recommendations. So far, most studies focused on a priori dietary indices, such as the Mediterranean Diet Score ${ }^{(30)}$, the Healthy Eating Index (HEI) ${ }^{(31)}$ and the Dietary Approaches to Stop Hypertension ${ }^{(15)}$, have found them to be associated with the risk of CKD. In contrast to the a priori approach, the a posteriori approach to identifying dietary patterns is derived from factor analysis, using data from dietary records or FFQ. Several studies have also reported the significant relationship between adherence to a posteriori dietary patterns and the risk of kidney function in the USA ${ }^{(17)}$, Europe ${ }^{(32)}$ and China ${ }^{(14)}$. In the China Health and Nutrition Survey, Shi et al. observed that the traditional Southern pattern characterized by high consumption of fruit, soya milk, egg and deep fried products was associated with a $50 \%$ decreased risk of $\mathrm{CKD}^{(14)}$. Thus, studies based on different cultures and populations may facilitate a better understanding of the importance of overall diet in the development of CKD.

In the present study, the traditional southern Chinese pattern was characterized by a high consumption of refined grains, vegetables, fruits, pickled vegetables, fish and shrimp, bacon and salted fish, salted and preserved eggs, milk, soyabean and its products, miscellaneous beans, fats and drinks. In our data, no significant association was observed between this pattern and the risk of CKD. Our findings are inconsistent with the previous study ${ }^{(14)}$, which reported that the traditional southern dietary pattern was positively associated with the risk of CKD. The complex nature of this pattern may explain the inconsistent findings to some extent. First, high consumption of refined grains has been found to increase the risk of diabetes, a risk factor for $\mathrm{CKD}^{(33)}$. Second, some foods (e.g. bacon and salted fish, salted and preserved eggs) have a high content of salt, which can increase the risk of hypertension, an important component of strategies to prevent $\mathrm{CKD}^{(1)}$. A recent study by Cheung et al. reported that high sodium intake might increase the risk of CKD in the Third National Health and Nutrition Examination Survey ${ }^{(9)}$. Third, drinks contain large amounts of fructose. Previous studies have shown that sugar consumption, especially in the form of fructose, is associated with an increased risk of kidney disease ${ }^{(34)}$. In contrast, vegetables and fruits also contain large amounts of dietary fibre. A previous study demonstrated that consumption of a diet rich in dietary fibre is inversely associated with the risk of $\mathrm{CKD}^{(27)}$. Besides, vegetables, fruits, fish and shrimp, milk, soyabean and its products in this pattern are rich in protective nutrients such as antioxidant vitamins, potassium, magnesium, calcium and phytochemicals. Yang et al. reported that these nutrients might affect kidney function and decrease the risk of $\mathrm{CKD}^{(35)}$. Meanwhile, the $n-3$ 
Table 3 Characteristics of the study participants by quartile (Q) categories of dietary pattern scores: Chinese adults aged 45-59 years ( $n$ 2004) from Hangzhou, Zhejiang Province, eastern China, June 2015-December 2016

\begin{tabular}{|c|c|c|c|c|c|c|c|c|c|c|c|c|c|c|c|}
\hline & \multicolumn{4}{|c|}{$\begin{array}{l}\text { Traditional southern Chinese dietary pattern } \\
\text { score }\end{array}$} & \multirow[b]{3}{*}{$P^{*}$} & \multicolumn{4}{|c|}{ Western dietary pattern score } & \multirow[b]{3}{*}{$P^{*}$} & \multicolumn{4}{|c|}{ Grains-vegetables dietary pattern score } & \multirow[b]{3}{*}{$P^{*}$} \\
\hline & \multicolumn{2}{|c|}{$\begin{array}{l}\text { Q1 (lowest) } \\
\quad(n 501)\end{array}$} & \multicolumn{2}{|c|}{$\begin{array}{l}\text { Q4 (highest) } \\
\quad \text { (n501) }\end{array}$} & & \multicolumn{2}{|c|}{$\begin{array}{l}\text { Q1 (lowest) } \\
\text { (n501) }\end{array}$} & \multicolumn{2}{|c|}{$\begin{array}{l}\text { Q4 (highest) } \\
\quad \text { (n501) }\end{array}$} & & \multicolumn{2}{|c|}{$\begin{array}{l}\text { Q1 (lowest) } \\
\quad(n 501)\end{array}$} & \multicolumn{2}{|c|}{$\begin{array}{l}\text { Q4 (highest) } \\
\text { (n501) }\end{array}$} & \\
\hline & Mean or $n$ & SD or $\%$ & Mean or $n$ & SD or $\%$ & & Mean or $n$ & SD or $\%$ & Mean or $n$ & SD or $\%$ & & Mean or $n$ & SD or $\%$ & Mean or $n$ & SD or $\%$ & \\
\hline Age (years) & $50 \cdot 9$ & 1.3 & 51.5 & 1.7 & $<0.05$ & $52 \cdot 4$ & 1.9 & $48 \cdot 6$ & 1.3 & $<0.001$ & $48 \cdot 3$ & $1 \cdot 7$ & 52.0 & 1.4 & $<0.001$ \\
\hline BMl $\left(\mathrm{kg} / \mathrm{m}^{2}\right)$ & 24.5 & $4 \cdot 3$ & 24.9 & $4 \cdot 1$ & 0.33 & $24 \cdot 1$ & 4.0 & $26 \cdot 6$ & $5 \cdot 3$ & $<0.01$ & $25 \cdot 7$ & 4.6 & 24.5 & 3.9 & $<0.05$ \\
\hline WC (cm) & $86 \cdot 7$ & 11.5 & $87 \cdot 2$ & 9.9 & 0.22 & $87 \cdot 2$ & 11.9 & $91 \cdot 1$ & $10 \cdot 9$ & $<0.01$ & 88.0 & 11.3 & 84.3 & $10 \cdot 1$ & $<0.01$ \\
\hline WHR & 0.86 & 0.08 & 0.85 & 0.08 & 0.34 & 0.85 & 0.08 & 0.90 & 0.07 & $<0.05$ & 0.86 & 0.06 & 0.83 & 0.08 & $<0.05$ \\
\hline Obesity & 81 & $16 \cdot 2$ & 70 & $14 \cdot 0$ & 0.33 & 68 & $13 \cdot 6$ & 106 & $21 \cdot 2$ & $<0.01$ & 89 & $17 \cdot 8$ & 53 & $10 \cdot 6$ & $<0.01$ \\
\hline Hypertension & 117 & $23 \cdot 4$ & 128 & $25 \cdot 5$ & 0.42 & 73 & $14 \cdot 6$ & 118 & $23 \cdot 6$ & $<0.001$ & 124 & 24.8 & 70 & $14 \cdot 0$ & $<0.001$ \\
\hline CKD & 95 & $19 \cdot 0$ & 121 & $24 \cdot 2$ & $<0.05$ & 98 & $19 \cdot 6$ & 151 & $30 \cdot 1$ & $<0.001$ & 135 & $26 \cdot 9$ & 76 & $15 \cdot 2$ & $<0.001$ \\
\hline Gender & & & & & 0.15 & & & & & $<0.001$ & & & & & $<0.001$ \\
\hline Male & 255 & $50 \cdot 9$ & 232 & $46 \cdot 3$ & & 247 & $49 \cdot 3$ & 337 & $67 \cdot 3$ & & 291 & $58 \cdot 1$ & 202 & $40 \cdot 3$ & \\
\hline Female & 246 & $49 \cdot 1$ & 269 & $53 \cdot 7$ & & 254 & $50 \cdot 7$ & 164 & $32 \cdot 7$ & & 210 & 41.9 & 299 & $59 \cdot 7$ & \\
\hline Smoking status & & & & & $<0.01$ & & & & & $<0.01$ & & & & & $<0.01$ \\
\hline Never & 368 & 73.4 & 327 & $65 \cdot 3$ & & 335 & $66 \cdot 9$ & 308 & 61.5 & & 387 & $77 \cdot 2$ & 429 & $85 \cdot 6$ & \\
\hline Current & 103 & $20 \cdot 6$ & 150 & 29.9 & & 123 & 24.5 & 168 & 33.5 & & 77 & 15.4 & 49 & $9 \cdot 8$ & \\
\hline Former & 30 & 6.0 & 24 & 4.8 & & 43 & 8.5 & 25 & 5.0 & & 37 & 7.4 & 23 & 4.6 & \\
\hline Education & & & & & 0.22 & & & & & 0.45 & & & & & 0.52 \\
\hline$<$ High school & 134 & $26 \cdot 8$ & 159 & $31 \cdot 7$ & & 101 & $20 \cdot 2$ & 104 & $20 \cdot 8$ & & 117 & 23.4 & 109 & 21.8 & \\
\hline High school & 174 & 34.7 & 162 & $32 \cdot 3$ & & 199 & $39 \cdot 7$ & 215 & 42.9 & & 176 & $35 \cdot 1$ & 166 & $33 \cdot 1$ & \\
\hline >High school & 193 & 38.5 & 180 & $36 \cdot 0$ & & 201 & $40 \cdot 1$ & 182 & $36 \cdot 3$ & & 208 & 41.5 & 226 & $45 \cdot 1$ & \\
\hline \multicolumn{3}{|c|}{ Monthly income per person (RMB) } & & & 0.06 & & & & & $<0.05$ & & & & & $<0.001$ \\
\hline$<2000$ & 134 & $26 \cdot 7$ & 107 & 21.4 & & 127 & $25 \cdot 3$ & 99 & $19 \cdot 8$ & & 179 & $35 \cdot 7$ & 90 & $18 \cdot 0$ & \\
\hline $2000-4000$ & 201 & $40 \cdot 1$ & 197 & $39 \cdot 3$ & & 178 & 35.5 & 169 & $33 \cdot 7$ & & 192 & 38.4 & 210 & 41.9 & \\
\hline$>4000$ & 166 & $33 \cdot 2$ & 197 & $39 \cdot 3$ & & 196 & $39 \cdot 2$ & 233 & $46 \cdot 5$ & & 130 & $25 \cdot 9$ & 201 & $40 \cdot 1$ & \\
\hline Physical activity & & & & & 0.16 & & & & & $<0.01$ & & & & & $<0.05$ \\
\hline Light & 407 & $81 \cdot 2$ & 389 & $77 \cdot 6$ & & 376 & $75 \cdot 0$ & 413 & $82 \cdot 4$ & & 420 & $83 \cdot 8$ & 388 & $77 \cdot 4$ & \\
\hline Moderate & 72 & 14.4 & 94 & $18 \cdot 8$ & & 101 & $20 \cdot 2$ & 78 & $15 \cdot 6$ & & 68 & $13 \cdot 6$ & 87 & $17 \cdot 4$ & \\
\hline Vigorous & 22 & 4.4 & 18 & 3.6 & & 24 & 4.8 & 10 & 2.0 & & 13 & 2.6 & 26 & $5 \cdot 2$ & \\
\hline Total energy (kJ/d) & 10469.6 & 851.9 & 10009.8 & $1083 \cdot 7$ & 0.14 & $9277 \cdot 2$ & $1036 \cdot 8$ & $11966 \cdot 2$ & $9920 \cdot 0$ & $<0.01$ & $10944 \cdot 1$ & $865 \cdot 7$ & 9391.4 & $900 \cdot 0$ & $<0.05$ \\
\hline Total energy (kcal/d) & $2502 \cdot 3$ & $203 \cdot 6$ & 2392.4 & $259 \cdot 0$ & 0.14 & $2217 \cdot 3$ & 247.8 & 2860.4 & 237.1 & $<0.01$ & $2615 \cdot 7$ & 206.9 & 2244.6 & $215 \cdot 1$ & $<0.05$ \\
\hline
\end{tabular}

WC, waist circumference; WHR, waist-to-hip rate; CKD, chronic kidney disease.

Continuous variables are presented as mean and standard deviation, categorical variables are presented as number and percentage.

${ }^{*} P$ value for continuous variables from the independent-samples $t$ test or the Mann-Whitney $U$ test, and for categorical variables from the $\chi^{2}$ test. 
Table 4 Major food consumption of the study participants by quartile $(Q)$ categories of dietary pattern scores: Chinese adults aged 45-59 years ( $n$ 2004) from Hangzhou, Zhejiang Province, eastern China, June 2015-December 2016

\begin{tabular}{|c|c|c|c|c|c|c|c|c|c|c|c|c|c|c|c|}
\hline & \multicolumn{4}{|c|}{$\begin{array}{l}\text { Traditional southern Chinese } \\
\text { dietary pattern score } \\
\end{array}$} & \multirow[b]{3}{*}{$P$} & \multicolumn{4}{|c|}{$\begin{array}{l}\text { Western dietary pattern } \\
\text { score }\end{array}$} & \multirow[b]{3}{*}{$P$} & \multicolumn{4}{|c|}{$\begin{array}{l}\text { Grains-vegetables dietary } \\
\text { pattern score }\end{array}$} & \multirow[b]{3}{*}{$P$} \\
\hline & \multicolumn{2}{|c|}{$\begin{array}{l}\text { Q1 (lowest) } \\
\quad(n 501)\end{array}$} & \multicolumn{2}{|c|}{$\begin{array}{l}\text { Q4 (highest) } \\
\quad(n \text { 501) }\end{array}$} & & \multicolumn{2}{|c|}{$\begin{array}{l}\text { Q1 (lowest) } \\
\quad(n 501)\end{array}$} & \multicolumn{2}{|c|}{$\begin{array}{l}\text { Q4 (highest) } \\
\text { (n 501) }\end{array}$} & & \multicolumn{2}{|c|}{$\begin{array}{l}\text { Q1 (lowest) } \\
\quad(n 501) \\
\end{array}$} & \multicolumn{2}{|c|}{$\begin{array}{l}\text { Q4 (highest) } \\
\quad(n \text { 501) } \\
\end{array}$} & \\
\hline & Mean & SD & Mean & SD & & Mean & SD & Mean & $\mathrm{SD}$ & & Mean & SD & Mean & SD & \\
\hline Refined grains & $282 \cdot 0$ & $149 \cdot 9$ & $365 \cdot 8$ & $188 \cdot 2$ & $<0.001$ & $295 \cdot 1$ & 164.9 & 349.2 & $156 \cdot 1$ & $<0.05$ & 282.7 & 171.4 & 273.9 & $140 \cdot 7$ & 0.26 \\
\hline Whole grain & $24 \cdot 1$ & 6.4 & 26.9 & 5.9 & & $20 \cdot 3$ & $7 \cdot 6$ & $16 \cdot 5$ & $7 \cdot 3$ & $<0.01$ & $19 \cdot 3$ & 8.1 & $36 \cdot 2$ & $10 \cdot 1$ & $<0.001$ \\
\hline Tubers & $17 \cdot 0$ & $12 \cdot 5$ & $20 \cdot 9$ & $11 \cdot 2$ & $<0.05$ & $21 \cdot 1$ & $11 \cdot 3$ & $19 \cdot 1$ & $13 \cdot 7$ & 0.51 & $18 \cdot 7$ & $10 \cdot 6$ & 28.9 & $11 \cdot 3$ & $<0.01$ \\
\hline Vegetables & 377.8 & 135.0 & $408 \cdot 2$ & $166 \cdot 9$ & 0.21 & 393.5 & $286 \cdot 2$ & 388.7 & $149 \cdot 5$ & 0.87 & $327 \cdot 3$ & $169 \cdot 1$ & $457 \cdot 8$ & $149 \cdot 6$ & $<0.001$ \\
\hline Fruits & $67 \cdot 3$ & 28.4 & $86 \cdot 2$ & 23.1 & $<0.01$ & 69.6 & 39.2 & $62 \cdot 4$ & 30.2 & 0.11 & 57.1 & $19 \cdot 3$ & 65.4 & 20.1 & $<0.05$ \\
\hline Red meat & $69 \cdot 6$ & $39 \cdot 2$ & 65.5 & $30 \cdot 2$ & 0.37 & $60 \cdot 0$ & $31 \cdot 6$ & 74.5 & $42 \cdot 2$ & $<0.001$ & $58 \cdot 6$ & $34 \cdot 1$ & 67.9 & $46 \cdot 2$ & $<0.05$ \\
\hline Fish and shrimp & $120 \cdot 9$ & 35.5 & 144.9 & $32 \cdot 8$ & $<0.01$ & 124.4 & 31.9 & $180 \cdot 6$ & $40 \cdot 3$ & $<0.001$ & $135 \cdot 6$ & 48.4 & 114.7 & $50 \cdot 8$ & $<0.05$ \\
\hline Eggs & $50 \cdot 3$ & $13 \cdot 2$ & 71.0 & $10 \cdot 6$ & $<0.001$ & $59 \cdot 0$ & $10 \cdot 8$ & 61.6 & $12 \cdot 8$ & 0.63 & 63.3 & 14.5 & $57 \cdot 8$ & $12 \cdot 4$ & $<0.05$ \\
\hline Drinks & $10 \cdot 6$ & 8.5 & 13.2 & $7 \cdot 1$ & 0.15 & $16 \cdot 7$ & $6 \cdot 7$ & 38.5 & $10 \cdot 4$ & $<0.001$ & 17.6 & 5.5 & $10 \cdot 0$ & 4.8 & $<0.05$ \\
\hline
\end{tabular}

Table 5 Multivariable-adjusted OR and $95 \% \mathrm{Cl}$ for chronic kidney disease across quartile $(\mathrm{Q})$ categories of dietary pattern scores: Chinese adults aged 45-59 years ( $n$ 2004) from Hangzhou, Zhejiang Province, eastern China, June 2015-December 2016

\begin{tabular}{|c|c|c|c|c|c|c|c|c|c|c|c|c|}
\hline & \multicolumn{3}{|c|}{$\begin{array}{l}\text { Traditional southern Chinese } \\
\text { pattern score }\end{array}$} & \multirow[b]{3}{*}{$P$} & \multicolumn{3}{|c|}{ Western pattern score } & \multicolumn{4}{|c|}{$\begin{array}{l}\text { Grains-vegetables pattern } \\
\text { score }\end{array}$} & \multirow[b]{3}{*}{$P$} \\
\hline & \multirow{2}{*}{$\frac{\mathrm{Q} 1}{\text { (reference) }}$} & \multicolumn{2}{|r|}{ Q4 } & & \multirow{2}{*}{$\frac{\mathrm{Q} 1}{\text { (reference) }}$} & \multicolumn{2}{|r|}{ Q4 } & \multirow[b]{2}{*}{$P$} & \multirow{2}{*}{$\frac{\mathrm{Q} 1}{\text { (reference) }}$} & \multicolumn{2}{|r|}{ Q4 } & \\
\hline & & OR & $95 \% \mathrm{Cl}$ & & & OR & $95 \% \mathrm{Cl}$ & & & OR & $95 \% \mathrm{Cl}$ & \\
\hline Model 1 & 1.00 & $1 \cdot 12$ & $0.94,1.38$ & 0.14 & 1.00 & $2 \cdot 27$ & $1.54,3.51$ & $<0.001$ & 1.00 & 0.36 & $0.26,0.48$ & $<0.001$ \\
\hline Model 2 & 1.00 & 1.03 & $0.81,1.28$ & 0.38 & 1.00 & $2 \cdot 02$ & $1 \cdot 35,3 \cdot 11$ & $<0.01$ & 1.00 & 0.65 & $0.58,0.72$ & $<0.05$ \\
\hline Model 3 & 1.00 & 0.94 & $0.78,1.11$ & 0.50 & 1.00 & 1.83 & $1.21,2 \cdot 81$ & $<0.05$ & 1.00 & 0.84 & $0.77,0.93$ & $<0.05$ \\
\hline
\end{tabular}

Q1, lowest quartile of dietary patterns (reference); Q4: highest quartile of dietary patterns.

Model 1: adjusted for gender and age (continuous).

Model 2: further adjusted for income (continuous), education level (<high school, high school, > high school), physical activity level (light, moderate, heavy), smoking status (never, current, former), BMI (continuous), type 2 diabetes mellitus (yes/no) and hypertension (yes/no).

Model 3: further adjusted for total energy intake.

PUFA abundant in fish and shrimp have also been reported to be associated with decreased risk of obesity and hypertension $^{(19)}$, which are known to be risk factors of CKD ${ }^{(36)}$. These possibilities cannot be excluded in the present study.

In the present study, we found a positive association between the Western dietary pattern and the risk of CKD. Our finding is line with a previous study ${ }^{(37)}$, which showed that the Western-style diet was a major risk factor for impaired kidney function and CKD. Several plausible explanations have been proposed to elucidate the detrimental association of this pattern with CKD risk. First, high intake of meat, containing large amounts of saturated fat and cholesterol, is strongly associated with the development of $\mathrm{CKD}^{(38)}$. Second, processed and cooked meat, seafood and fast foods in the Western pattern often contain a lot of salt. As already discussed ${ }^{(1)}$, high salt consumption is positively associated with the development of CKD. Third, in our previous study, the Western dietary pattern was found to be significantly associated with increased risk of obesity, hypertension and T2DM, and all of these factors have been documented to be important risk factors for $\operatorname{CKD}^{(1,27)}$.
The grains-vegetables dietary pattern was characterized by high consumption of whole grains, tubers, vegetables, mushrooms, vegetable oil, nuts, honey and tea. In our analyses, we observed an inverse association between the grains-vegetables pattern and the risk of CKD. These results are in agreement with prior studies ${ }^{(17,32,39)}$ which demonstrated that a diet with higher intakes of whole grains, vegetables and fruits is inversely associated with the risk of CKD. The possible mechanism for the protective effect of the grains-vegetables pattern on CKD may be due to the content of whole grains, vegetables, mushrooms and tea, which are rich in fibres, folate and antioxidants (e.g. vitamin $\mathrm{C}$, vitamin $\mathrm{E}$ and other carotenoid compounds). First, as mentioned above, high consumption of dietary fibre can play a protective role in the risk of CKD by lowering the levels of inflammatory markers including IL6, total homocysteine and C-reactive protein ${ }^{(27,40)}$. Second, high consumption of vegetables, containing amounts of folate, is associated with a decreased risk of $\mathrm{CKD}^{(13,35)}$. Carney reported that after a median follow-up of 4.4 years, combined daily treatment with 
enalapril (10 mg) and folic acid $(0.8 \mathrm{mg})$, compared with daily enalapril alone, reduced the risk of CKD progression by $21 \%$ and the rate of eGFR decline by $10 \%$ among 15104 Chinese adults (aged 45-75 years) with hypertension and eGFR $>30 \mathrm{ml} / \mathrm{min}$ per $1.73 \mathrm{~m}^{2}$ at baseline ${ }^{(41)}$. Finally, vegetables and tea that provide large amounts of antioxidant substances (e.g. vitamin C, vitamin E, other carotenoid compounds) may contribute to reducing the risk of $\mathrm{CKD}^{(35)}$. A recent study by Jankowska et al. investigated the status of dietary intake of vitamins in patients with $\mathrm{CKD}$, reporting an inverse relationship between vitamin intake and risk of $\mathrm{CKD}^{(10)}$.

\section{Strengths and limitations}

The present study has both strengths and limitations. First, to our knowledge, it is the first study to examine the association between empirically derived dietary patterns and the risk of CKD in a middle-aged Chinese population. Second, dietary information was collected by well-trained dietitians using a validated semi-quantitative FFQ. This ensured that the dietary data we collected are accurate. Third, the present findings are reliable because we have controlled for several potential confounders in the final analysis. Nevertheless, some limitations of the present study need to be acknowledged. First, because of the cross-sectional design of the study, we could not assess the causal association between dietary patterns and the risk of CKD. Therefore, further prospective studies are needed to confirm this finding. Second, statistical methods used to derive dietary patterns, including food grouping and principal component analysis, involve several subjective decisions $^{(42)}$. Third, because of the nature of the selfreporting questionnaire, recall bias exists and the estimates of food frequency may be not accurate in FFQ. Fourth, the study participants with previous diagnosed CKD may have been advised to change their diet as part of a strategy to slow the progression of the disease, which in turn could affect our findings. Finally, participants in our study were predominately recruited in the city of Hangzhou and are not a random sample of the general population. Therefore, the present findings may not be extrapolated to the general population.

\section{Conclusions}

In conclusion, our study suggested that the Western pattern was associated with higher risk, whereas the grainsvegetables pattern was associated with lower risk of CKD. These findings provide further insight to better understand the association between dietary patterns and CKD risk. Given the cross-sectional nature of our study, future prospective studies are warranted to confirm the causal association between dietary patterns and the risk of CKD.

\section{Acknowledgements}

Acknowledgements: The authors thank all participants from the School of Medicine, Huainan Union University and the Department of Nutrition, Zhejiang Hospital for their assistance and support. They also acknowledge staff of the Medical Center for Physical Examination, Zhejiang Hospital for their important contribution to collection of data in this study. Financial support: This study was supported by Natural Science Foundation of Zhejiang (grant number LY17H030008) and Natural Science Research Project of Anhui University (grant number KJ2019A1003). The Natural Science Foundation of Zhejiang and Natural Science Research Project of Anhui University had no role in the design, analysis or writing of this article. Conflict of interest: The authors declare that there is no conflict of interests. Authorship: S.-S.X. and L.S. contributed to the study design. J.H. and Y.-Q.H. performed the statistical analysis for the manuscript and S.-S.X. drafted the paper. All authors contributed to a critical review of the manuscript during the writing process. Ethics of human subjectparticipation: This study was conducted according to the guidelines laid down in the Declaration of Helsinki and all procedures involving research study participants were approved by the institutional review and ethics committee of Zhejiang Hospital. Written informed consent was obtained from all participants.

\section{Supplementary material}

To view supplementary material for this article, please visit https://doi.org/10.1017/S1368980019002805

\section{References}

1. Jha V, Garcia-Garcia G, Iseki K et al. (2013) Chronic kidney disease: global dimension and perspectives. Lancet 382, 260-272.

2. Ayodele OE \& Alebiosu CO (2010) Burden of chronic kidney disease: an international perspective. Adv Chronic Kidney Dis 17, 215-224.

3. Coresh J, Selvin E, Stevens LA et al. (2007) Prevalence of chronic kidney disease in the United States. JAMA 298, 2038-2047.

4. Wen CP, Cheng TY, Tsai MK et al. (2008) All-cause mortality attributable to chronic kidney disease: a prospective cohort study based on 462293 adults in Taiwan. Lancet 371, 2173-2182.

5. Zhang L, Wang F, Wang L et al. (2012) Prevalence of chronic kidney disease in China: a cross-sectional survey. Lancet 379, 815-822.

6. Banerjee T, Liu Y \& Crews DC (2016) Dietary patterns and CKD progression. Blood Purif 41, 117-122.

7. Khatri M, Moon YP, Scarmeas N et al. (2014) The association between a Mediterranean-style diet and kidney function in the Northern Manhattan Study cohort. Clin J Am Soc Nephrol 9, 1868-1875.

8. Strippoli GF, Craig JC, Rochtchina E et al. (2011) Fluid and nutrient intake and risk of chronic kidney disease. Nephrology (Carlton) 16, 326-334. 
9. Cheung CL, Sahni S, Cheung BM et al. (2015) Vitamin K intake and mortality in people with chronic kidney disease from NHANES III. Clin Nutr 34, 235-240.

10. Jankowska M, Szupryczyńska N, Dębska-Ślizień A et al. (2016) Dietary intake of vitamins in different options of treatment in chronic kidney disease: is there a deficiency? Transplant Proc 48, 1427-1430.

11. Hu FB (2002) Dietary pattern analysis: a new direction in nutritional epidemiology. Curr Opin Lipidol 13, 3-9.

12. Shu L, Shen XM, Li C et al. (2017) Dietary patterns are associated with type 2 diabetes mellitus among middle-aged adults in Zhejiang Province, China. Nutr J 16, 81.

13. Mazidi M, Gao HK \& Kengne AP (2018) Food patterns are associated with likelihood of CKD in US adults. Sci Rep $\mathbf{8}$, 10696.

14. Shi Z, Taylor AW, Riley M et al. (2018) Association between dietary patterns, cadmium intake and chronic kidney disease among adults. Clin Nutr 37, 276-284.

15. Lee HS, Lee KB, Hyun YY et al. (2017) DASH dietary pattern and chronic kidney disease in elderly Korean adults. Eur J Clin Nutr 71, 755-761.

16. Wai SN, Kelly JT, Johnson DW et al. (2017) Dietary patterns and clinical outcomes in chronic kidney disease: the CKD.QLD Nutrition Study. J Ren Nutr 27, 175-182.

17. Lin J, Fung TT, Hu FB et al. (2011) Association of dietary patterns with albuminuria and kidney function decline in older white women: a subgroup analysis from the Nurses' Health Study. Am J Kidney Dis 57, 245-254.

18. Gutiérrez OM, Muntner P, Rizk DV et al. (2014) Dietary patterns and risk of death and progression to ESRD in individuals with CKD: a cohort study. Am J Kidney Dis $\mathbf{6 4}$, 204-213.

19. Zheng PF, Shu L, Zhang XY et al. (2016) Association between dietary patterns and the risk of hypertension among Chinese: a cross-sectional study. Nutrients $\mathbf{8}, 239$.

20. Wang CJ, Yang TF, Wang GS et al. (2018) Association between dietary patterns and depressive symptoms among middle-aged adults in China in 2016-2017. Psychiatry Res 260, 123-129.

21. Esmaillzadeh A, Kimiaqar M, Mehrabi Y et al. (2007) Dietary patterns, insulin resistance, and prevalence of the metabolic syndrome in women. Am J Clin Nutr 85, 910-918.

22. Yang CQ, Shu L, Wang S et al. (2015) Dietary patterns modulate the risk of non-alcoholic fatty liver disease in Chinese adults. Nutrients 7, 4778-4791.

23. Chobanian AV, Bakris GL, Black HR et al. (2003) The seventh report of the Joint National Committee on Prevention, Detection, Evaluation, and Treatment of High Blood Pressure: the JNC 7 report. JAMA 289, 2560-2572.

24. Chavers BM, Simonson J \& Michael AF (1984) A solid phase fluorescent immunoassay for the measurement of human urinary albumin. Kidney Int 25, 576-578.

25. Wang HJ, Wang ZH, Yu WT et al. (2008) Changes of waist circumference distribution and the prevalence of abdominal adiposity among Chinese adults from 1993 to 2006. Zhonghua Liu Xing Bing Xue Za Zhi 29, 953-958.
26. Yu XL, Shu L, Shen XM et al. (2017) Gender difference on the relationship between hyperuricemia and nonalcoholic fatty liver disease among Chinese: an observational study. Medicine (Baltimore) 96, e8164.

27. Farhadnejad H, Asghari G, Mirmiran P et al. (2016) Micronutrient intakes and incidence of chronic kidney disease in adults: Tehran Lipid and Glucose Study. Nutrients $\mathbf{8}, 217$.

28. Lee SA, Cai H, Yang G et al. (2010) Dietary patterns and blood pressure among middle-aged and elderly Chinese men in Shanghai. Br J Nutr 104, 265-275.

29. Reddy KS \& Katan MB (2004) Diet, nutrition and the prevention of hypertension and cardiovascular diseases. Public Health Nutr 7, 167-186.

30. Chrysohoou C, Panagiotakos DB, Pitsavos C et al. (2010) Adherence to the Mediterranean diet is associated with renal function among healthy adults: the ATTICA study. J Ren Nutr 20, 176-184.

31. Gopinath B, Harris DC, Flood VM et al. (2013) A better diet quality is associated with a reduced likelihood of CKD in older adults. Nutr Metab Cardiovasc Dis 23, 937-943.

32. Nettleton JA, Steffen LM, Palmas W et al. (2008) Associations between microalbuminuria and animal foods, plant foods, and dietary patterns in the Multiethnic Study of Atherosclerosis. Am J Clin Nutr 87, 1825-1836.

33. Hu EA, Pan A, Malik V et al. (2012) White rice consumption and risk of type 2 diabetes: meta-analysis and systematic review. BMJ 344, e1454.

34. Karalius VP \& Shoham DA (2013) Dietary sugar and artificial sweetener intake and chronic kidney disease: a review. Adv Chronic Kidney Dis 20, 157-164.

35. Yang M, Fox CH, Vassalotti J et al. (2011) Complications of progression of CKD. Adv Chronic Kidney Dis 18, $400-405$

36. Huang X, Jiménez-Moleón JJ, Lindholm B et al. (2013) Mediterranean diet, kidney function, and mortality in men with CKD. Clin J Am Soc Nephrol 8, 1548-1555.

37. Odermatt A (2011) The Western-style diet: a major risk factor for impaired kidney function and chronic kidney disease. $\mathrm{Am}$ J Physiol Renal Physiol 301, F919-931.

38. Lin J, Hu FB \& Curhan GC (2010) Associations of diet with albuminuria and kidney function decline. Clin J Am Soc Nephrol 5, 836-843.

39. Hsu CC, Jhang HR, Chang WT et al. (2014) Associations between dietary patterns and kidney function indicators in type 2 diabetes. Clin Nutr 33, 98-105.

40. Wannamethee SG, Lowe GD, Rumley A et al. (2006) Associations of vitamin C status, fruit and vegetable intakes, and markers of inflammation and hemostasis. Am J Clin Nutr 83, 567-574.

41. Carney EF (2016) Nutrition: folic acid delays CKD progression. Nat Rev Nephrol 12, 580.

42. Htun NC, Suga H, Imai S et al. (2018) Dietary pattern and its association with blood pressure and blood lipid profiles among Japanese adults in the 2012 Japan National Health and Nutrition Survey. Asia Pac J Clin Nutr 27, 1048-1061. 